\title{
Design and Evaluation of a Smartphone-Based Application to Manage the Treatment of People with Heart Failure
}

\section{ART ICLE INF O}

\section{Article Type}

Original Research

\section{Authors}

Davoudi Kongsofla M. ${ }^{1} M S c$, Najafi Ghezeljeh T. ${ }^{* 2} P h D$, Saeidi Shahrivar A. ${ }^{1} M S c$, Peyravi H. ${ }^{2} P h D$,

Kiaroosta $\mathrm{N}^{3} \mathrm{MSC}$

How to cite this article
Davoudi Kongsofla M, Najafi, Gh-
ezeljeh T, Saeidi Shahrivar A
Peyravi H, Kiaroosta N. Design and
Evaluation of a Smartphone-
Based Application to Manage the
Treatment of People with Heart
Failure. Iranian Journal of War
$\&$ Public Health
$2019 ; 11(3): 125-131$.

\section{A B S T R A C T}

Aims Patients with heart failure need training and support regarding the drug diet, how to use medications, diet, activities, and appropriate action when symptoms occur. The aim of this study was to design and evaluate a smartphone-based application to manage the treatment of people with heart failure.

Materials \& Methods In this developmental-applied study with the software design approach, from December 2017 to July 2018, in order to determine the capabilities and data needed for the application, searches in databases, review the texts, and health experts' opinion were used and the program was designed under the Android operating system with a dashboard under Windows. The program in the treatment management was evaluated based on the Smartphone Application Scale (MARS), the performance score of the Institute of Health Informatics (IMS), and the compliance with the Guide to the American Heart Failure Association (HFSA) by 20 patients with heart failure and their family, health staff, and programmers.

Findings The smartphone-based application with the mean score of $10.70 \pm 0.86$ in the MARS scale, the mean score of $9.00 \pm 0.87$ in the IMS scale, and the mean score of $7.7 \pm 0.79$ according to HFSA criteria was evaluated as desirable.

Conclusion The use of a smartphone-based application can be useful in treatment management and self-care of patients with heart failure.

Keywords Heart failure; Smartphone; Electronic health; Mobile health; Self-care
${ }^{1}$ Janbazan Medical \& Engineering Research Center (JMERC), Tehran, Iran

2"Nursing Care Research Center" and "Emergency \& Critical Care Nursing Department, Nursing \& Midwifery Faculty", Iran University of Medical Sciences, Tehran, Iran ${ }^{3}$ Emergency \& Critical Care Nursing Department, Nursing \& Midwifery Faculty, Iran University of Medical Sciences, Tehran, Iran

\section{*Correspondence}

Address: Nursing \& Midwifery Faculty, Rashid Yasemi Street, Valiasr Street, Tehran, Iran. Postal Code: 1996713883

Phone: +98 (21) 43651714

Fax: +98 (21) 88201978

najafi.t@iums.ac.ir

\section{Article History}

Received: September 18, 2018

Accepted: February 16, 2019

ePublished: July 21, 2019

\section{I T A T I O N L IN K S}

[1] Braunwald's heart disease: a textbook ... [2] Understanding population cardiovascular health ... [3] Heart disease and stroke statistics-2014 update: a report from ... [4] Application of the health belief model in promotion ... [5] The influence of a postdischarge intervention ... [6] Impact of medication nonadherence on ... [7] Medication adherence interventions improve heart ... [8] A multisite randomized trial of a single- session ... [9] HFSA 2010 comprehensive heart ... [10] Improving treatment adherence in heart failure: a ... [11] Gerontechnologies for older patients with heart failure ... [12] The effectiveness of mobile-health technologies to ... [13] Supporting cancer patients in illness management ... [14] Quantifying the body: monitoring and measuring ... [15] Relation between perceived social support and health ... [16] The effect of presence (lecture) and nonpresence ... [17] Provide telemedicine services to ... [18] Mineralocorticoid receptor antagonists for heart... [19] A framework to assist health professionals in recommending ... [20] Review and analysis of existing mobile phone apps to support ... [21] The role of mobile technologies in health care ... [22] Economic impact assessment from the use of a mobile app ... [23] Obesity surgery smartphone apps ... [24] A systematic review of smartphone applications ... [25] Smartphone-based home care model improved use of ... [26] Introduction and use of an education-notification application ... [27] Mobile app rating scale: a new tool for assessing ... [28] Patient apps for improved healthcare: from novelty ... [29] Metabolomic profiling identifies novel circulating biomarkers of mitochondrial ... [30] Ergonomic evaluation and design of a mobile application ... [31] Mobile phone-based telemonitoring for heart failure ... [32] Effect of educational program on quality of life of ... [33] The development and acceptability of a mobile application for tracking ... [34] Smartphone and tablet self management apps ... [35] Surgical site infections: epidemiology and microbiological ... 
كاهش يذيرش مجدد و ارتقاى كيفيت زندگى و كاهش مرك،

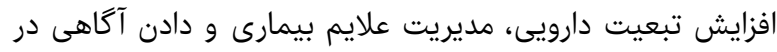

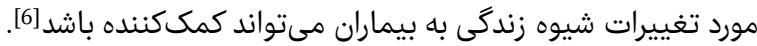

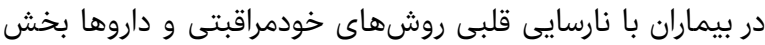

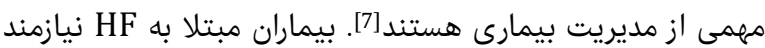

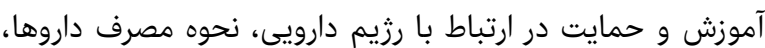

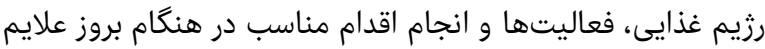

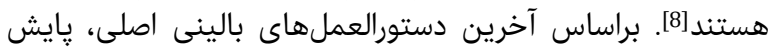

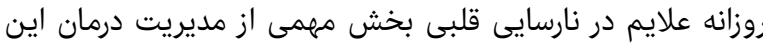

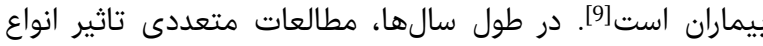

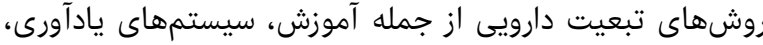

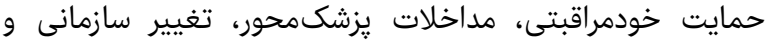

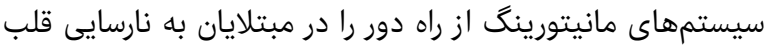

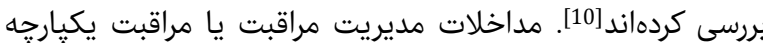

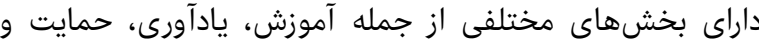

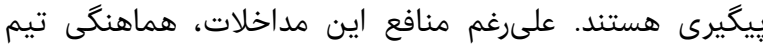

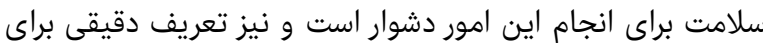

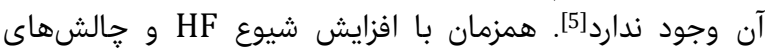

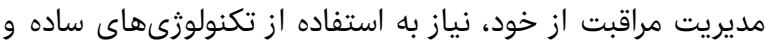

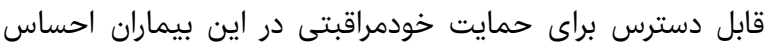

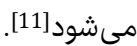

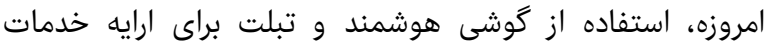

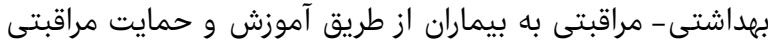

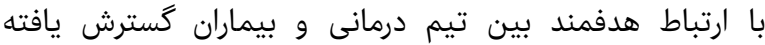

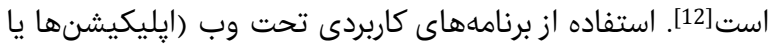

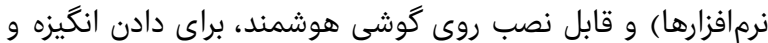

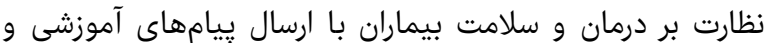

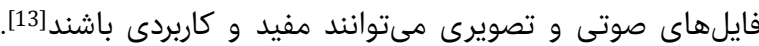

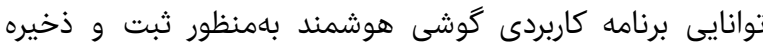

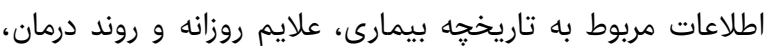

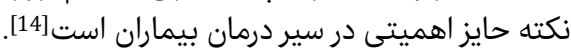

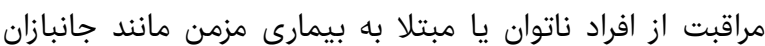

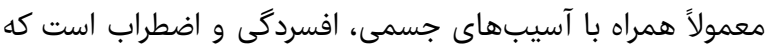

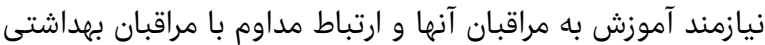

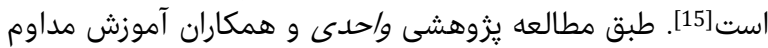

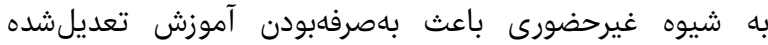

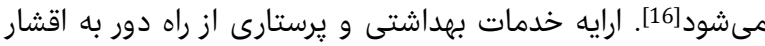

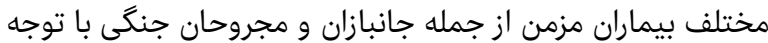

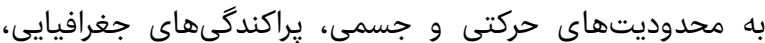

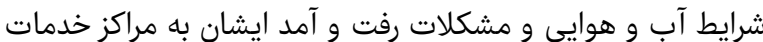

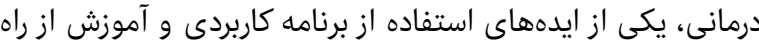

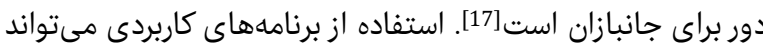

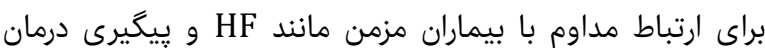

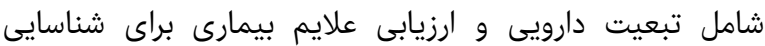

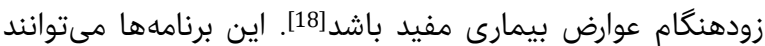

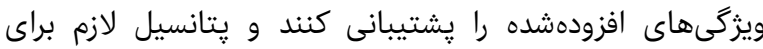

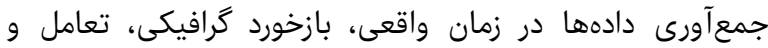

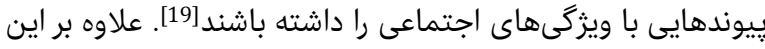

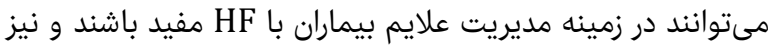

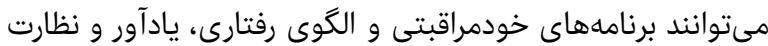

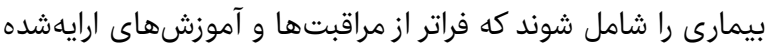

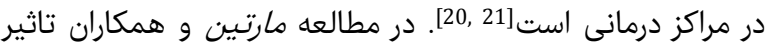

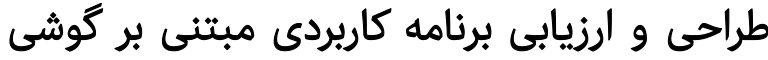
هوشمند براى مديريت درمان افراد برام بارئ نارسايى برديى

\section{MSc محبوبه داودى كنگسفلى \\ مركز تحقيقات مهندسى و علوم علوم يزشكى جانبازان، تهران، ايران

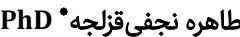

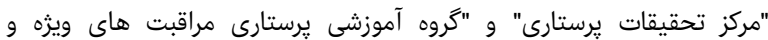

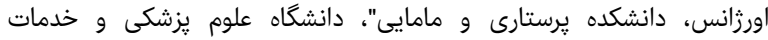

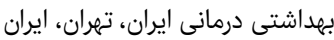

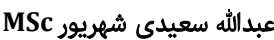

مركز تحقيقات مهندسى و مُهيور علوم يزشكى جانبازان، تهران، ايران

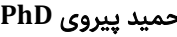

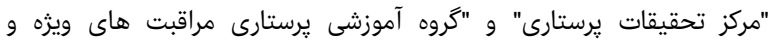

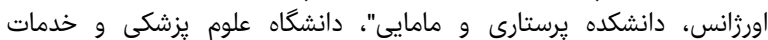

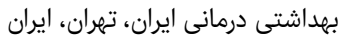

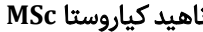

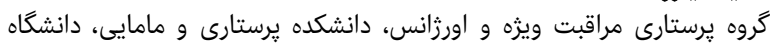
علوم يزشكى و خدمات بهداشتى درمانى ايران، تهران، ايران

جكيده

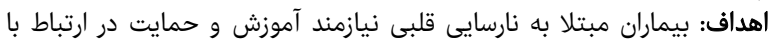

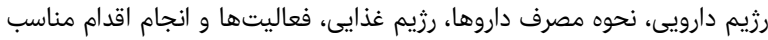

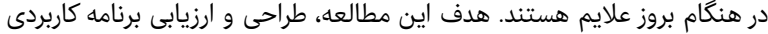

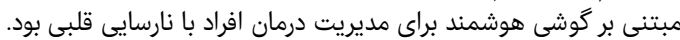

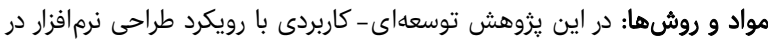

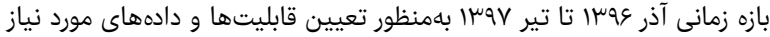

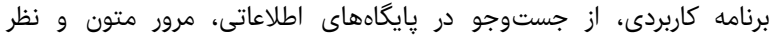

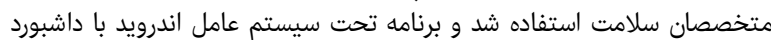

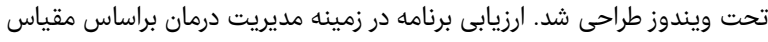

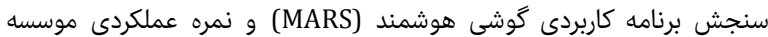

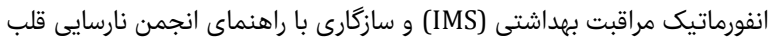

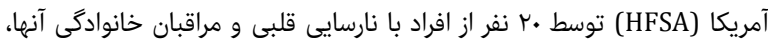

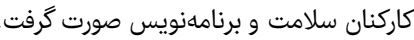

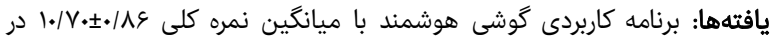

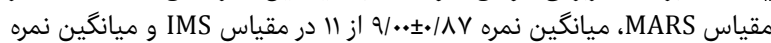

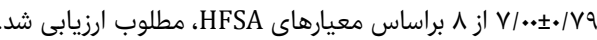

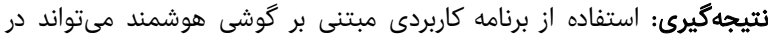

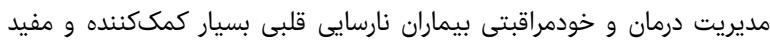
باشد. كليدواثهها: نارسايى قلبى، گَوشى هوشمند، سلامت الكترونيك، سلامت همراه،

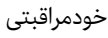

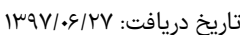

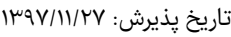

najafi.t@iums.ac.ir :نويسنده مسئول: تونيخ:

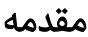

نارسايى قلبى (HF) سندروم بالينى يِيجيده و يِيشروندهاى است

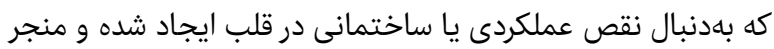

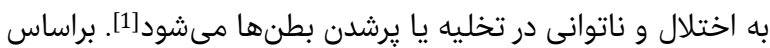

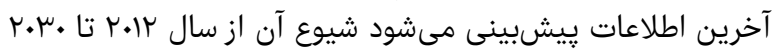

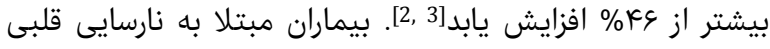

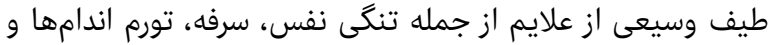

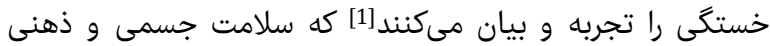

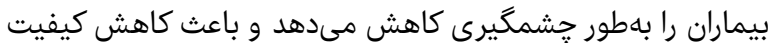

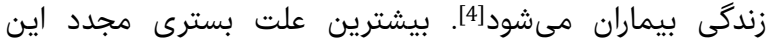

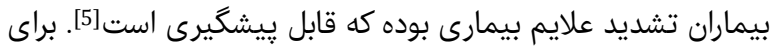




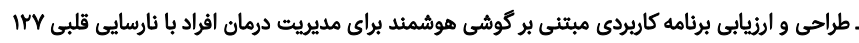

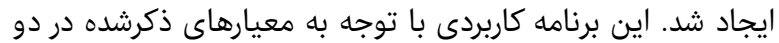

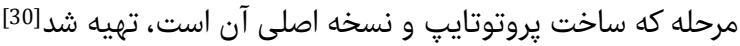

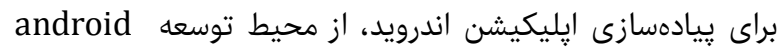

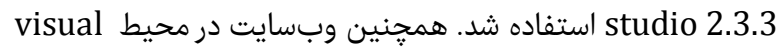

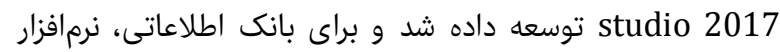

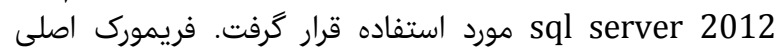
وبسايت ASP .Net MVC و فريمورى دسترسى به دادهادهاى

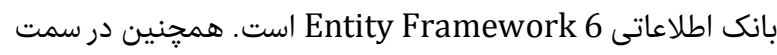
كلاينت از كتابخانههاى جاوا اسكرييتى JQ Juery UI ،JQuery JqGrid

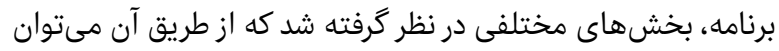

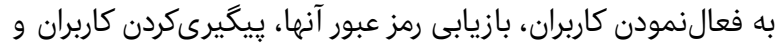

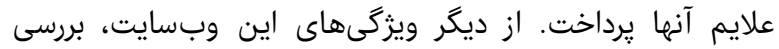

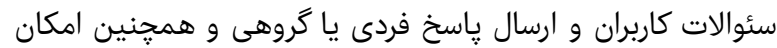

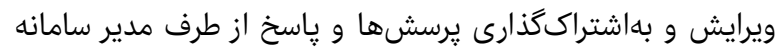

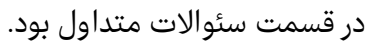

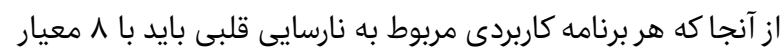

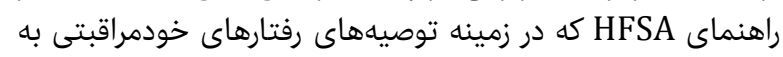

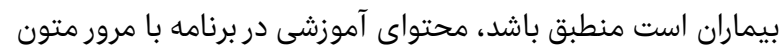

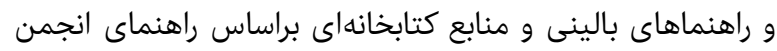

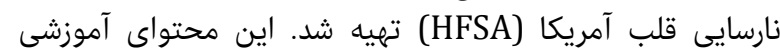

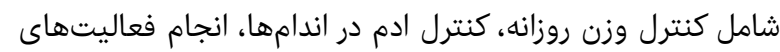

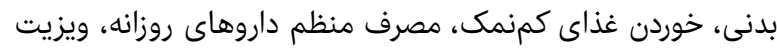

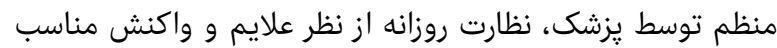

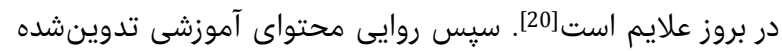

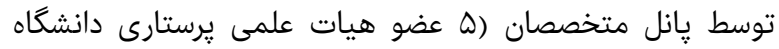

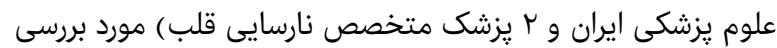
و تاييد قرار گرفت.

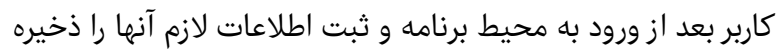

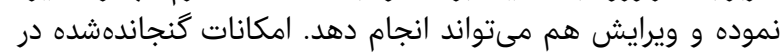

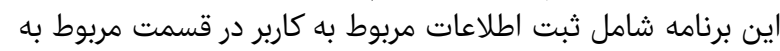

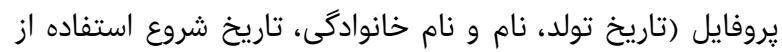

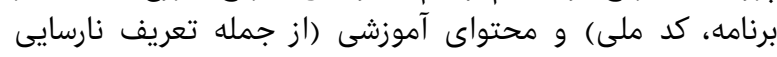

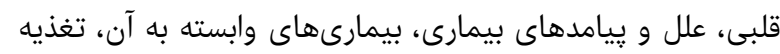

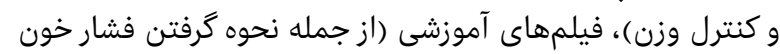

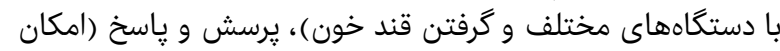

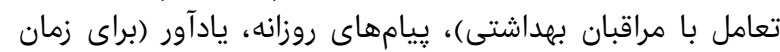

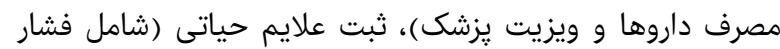

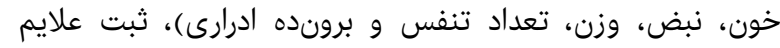

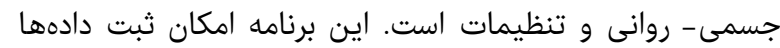

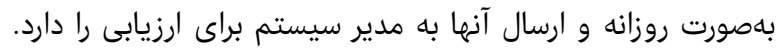

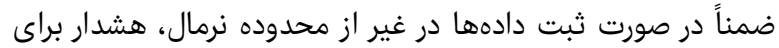

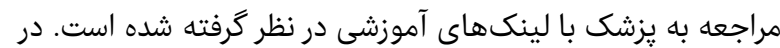

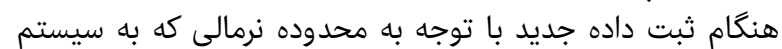

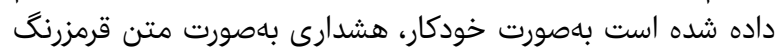

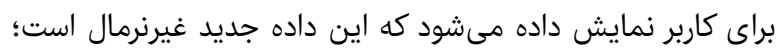

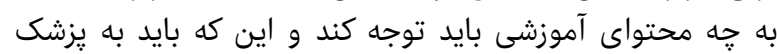

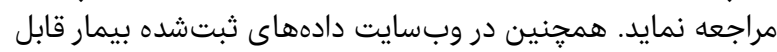

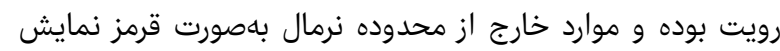

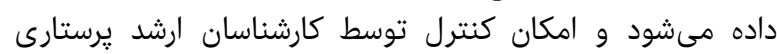

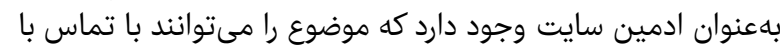

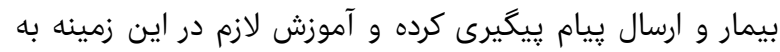

اقتصادى استفاده از برنامههاى كاربردى مبتنى بر گوشى هوشمند

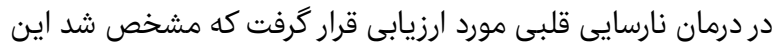

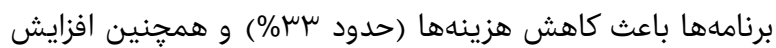
كيفيت زندگى بيماران و افزايش كارآيى سيسته

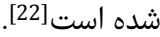

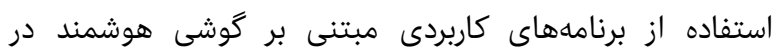

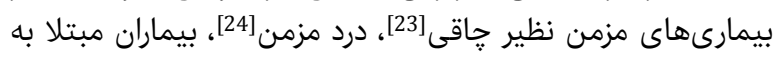

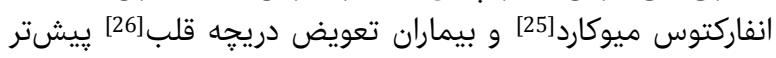

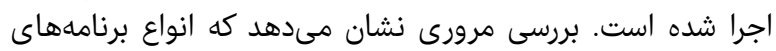

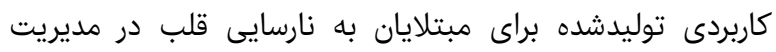

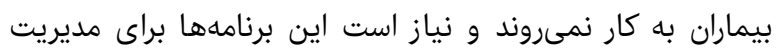

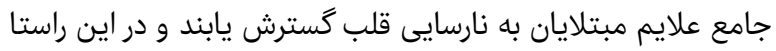

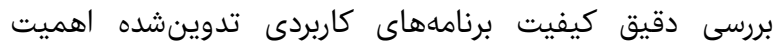

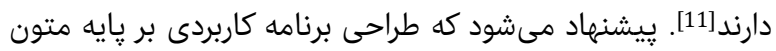

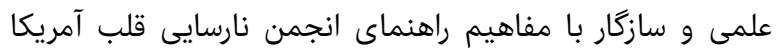

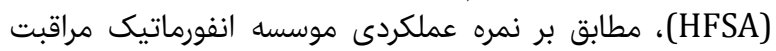

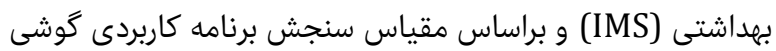

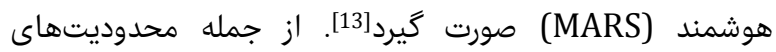

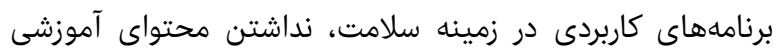

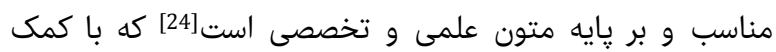

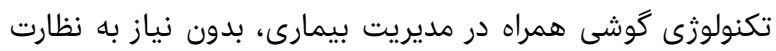

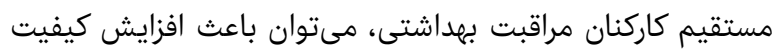

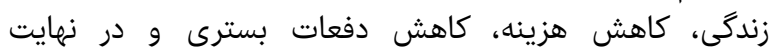

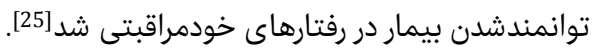

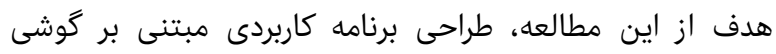

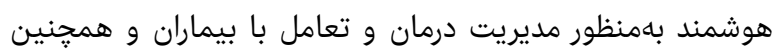
آموزش بيماران با نارسايى قلبى بود مدريت دوران

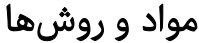

اين يزوهش از نو نوع توسعهاى ـ كاربردى با رويكرد طراحى نرمافزار

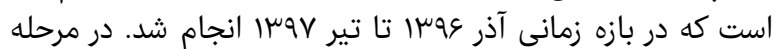

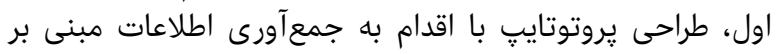

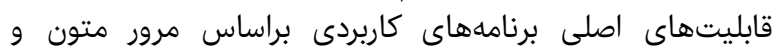

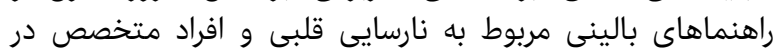

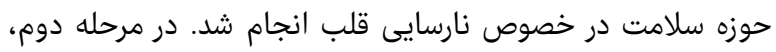

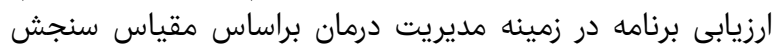

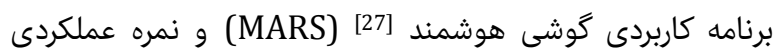

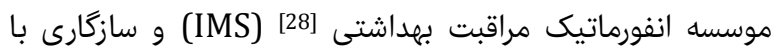

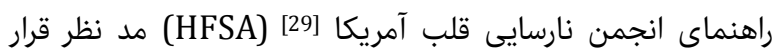

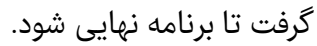

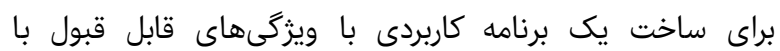

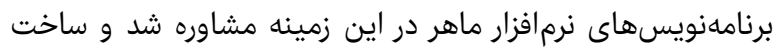

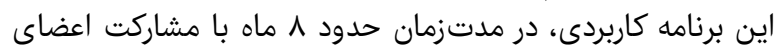

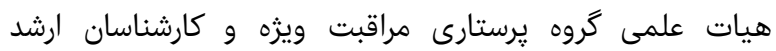

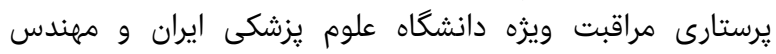

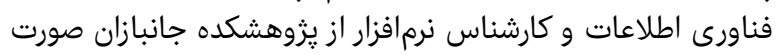

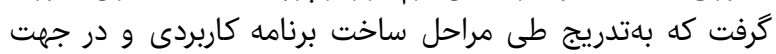

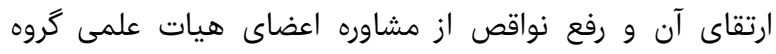

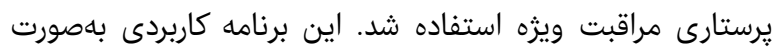

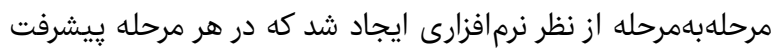

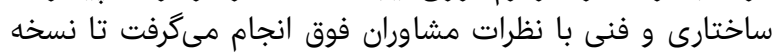

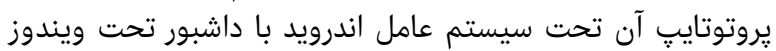


مطالعه حاضر روايى محتوايى اين ابزار توسط يانل متخصصان

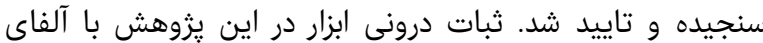

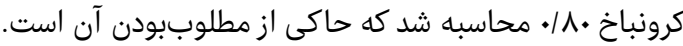

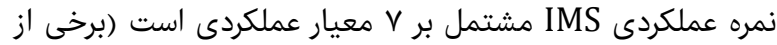

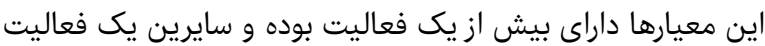

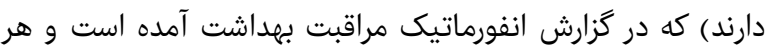

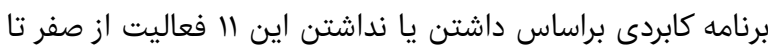

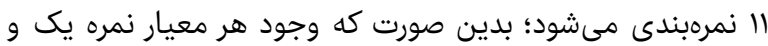

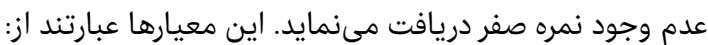

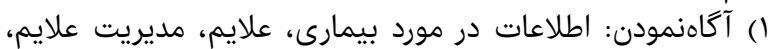

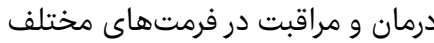

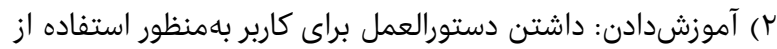

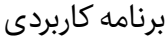

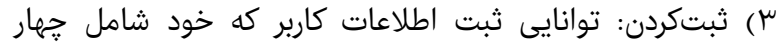

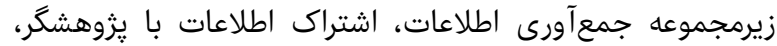

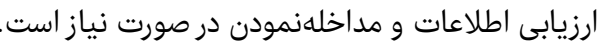

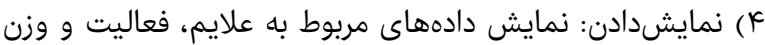

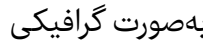
ه) راهنما: ارايه راهنمايى براساس دادههاى واردشيده توسط كاربر و

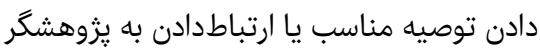

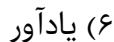

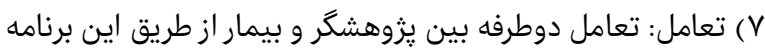

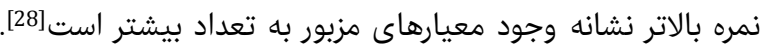

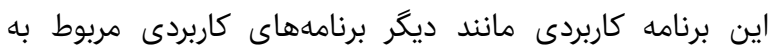

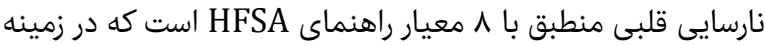

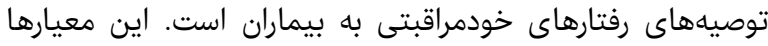

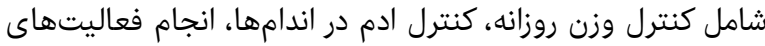

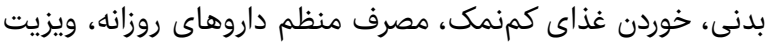

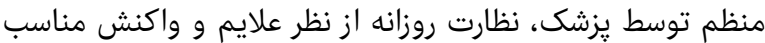

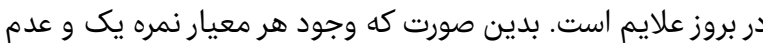

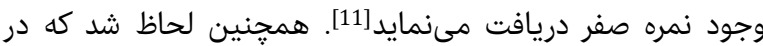

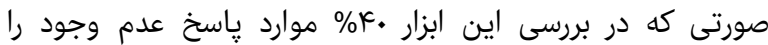

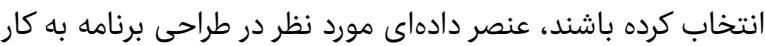

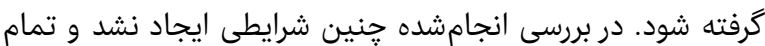

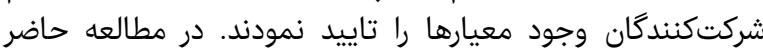

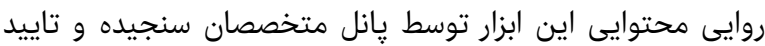

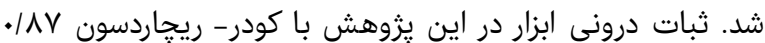

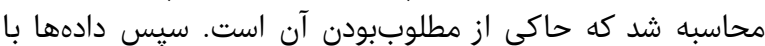

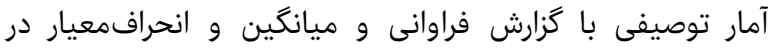

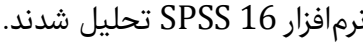

يافتهها

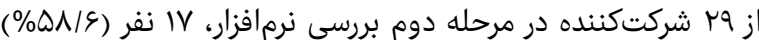

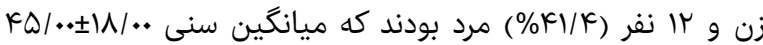

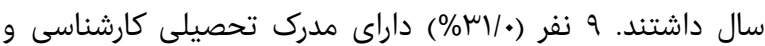

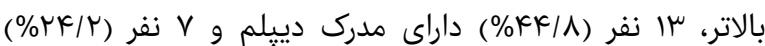

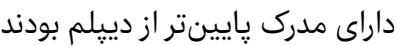

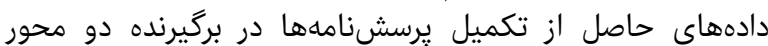

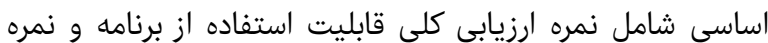

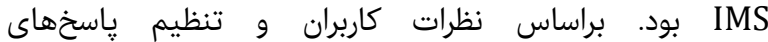

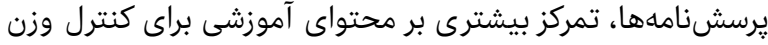

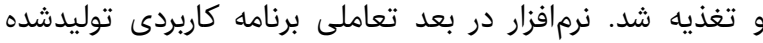

بيمار داده شود. ضمناً در برنامه كاربرى هر شخص اردار امكان رويت

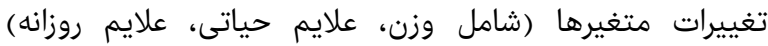

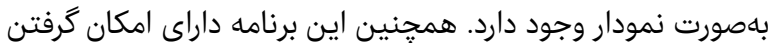

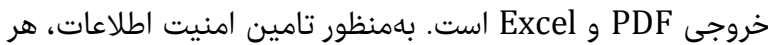

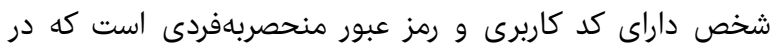

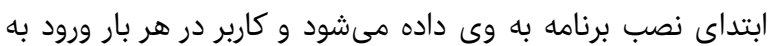

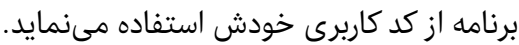

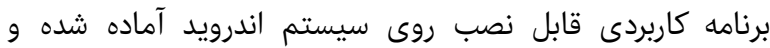

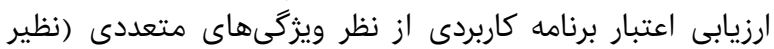

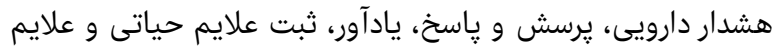

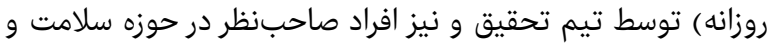

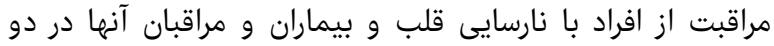

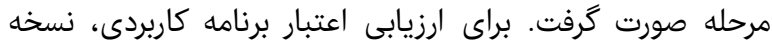

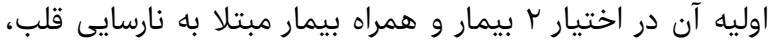

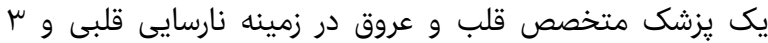

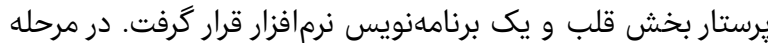

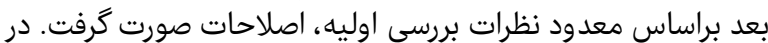

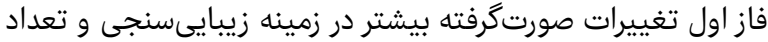

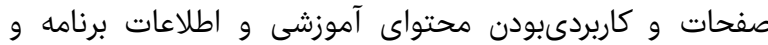

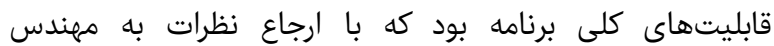

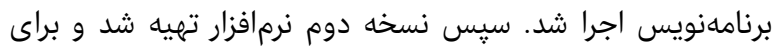

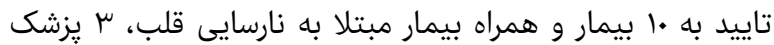

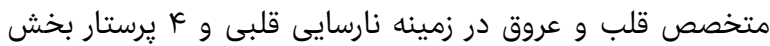

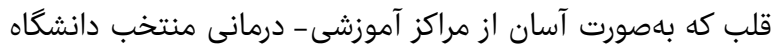

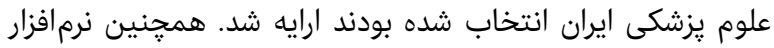

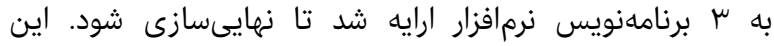

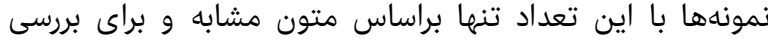

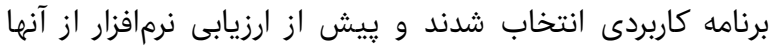

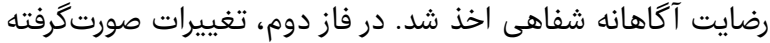

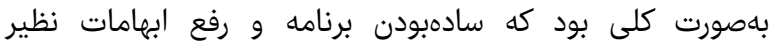

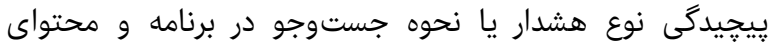

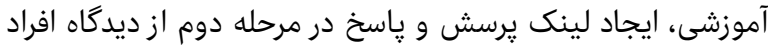

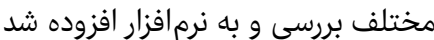

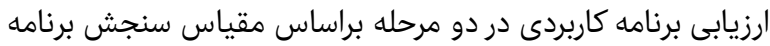

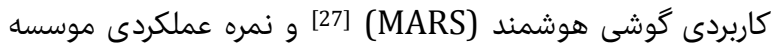

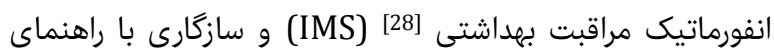

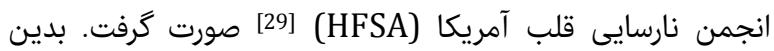

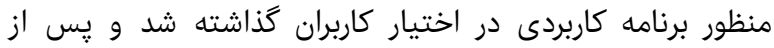

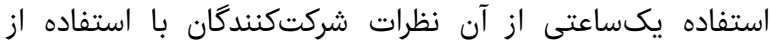

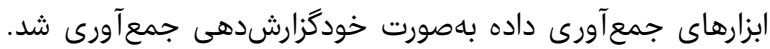

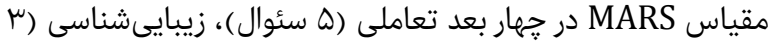

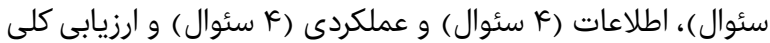

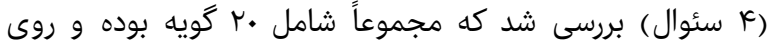

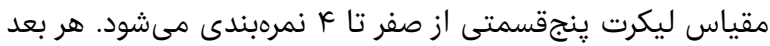

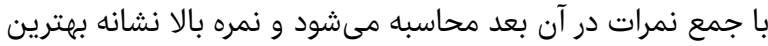

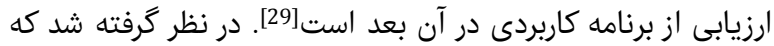

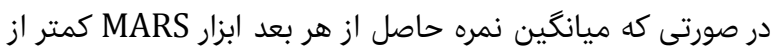

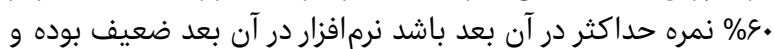

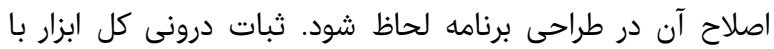

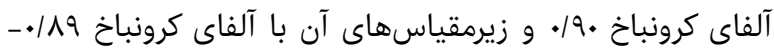

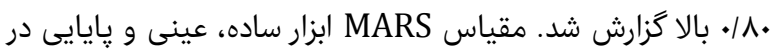

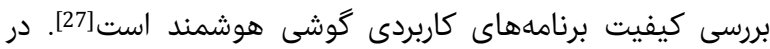




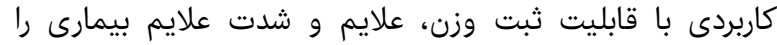

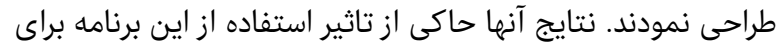

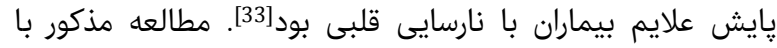

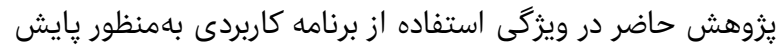

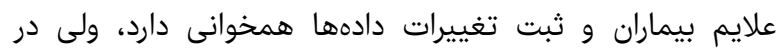

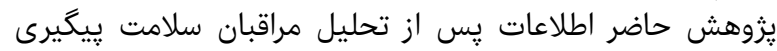

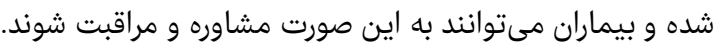

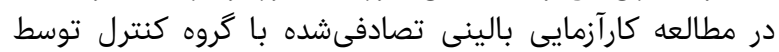

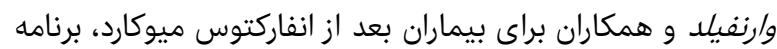

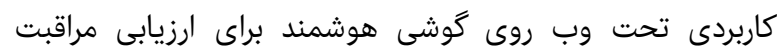

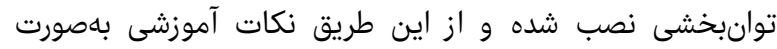

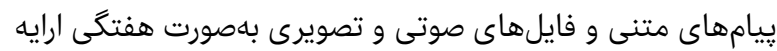

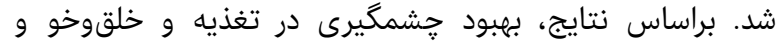

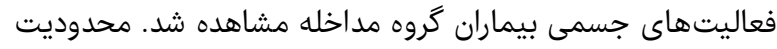

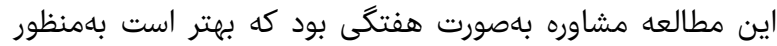

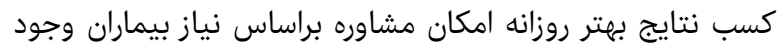

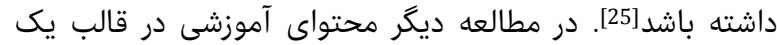

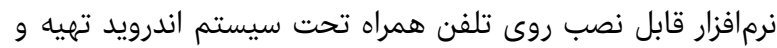

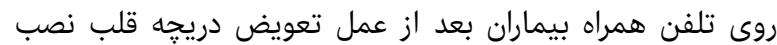

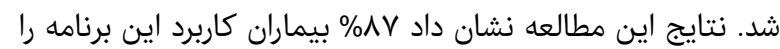

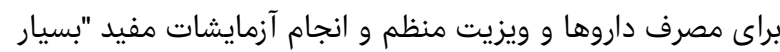

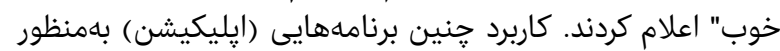

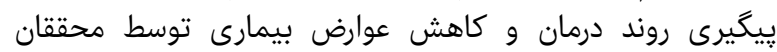

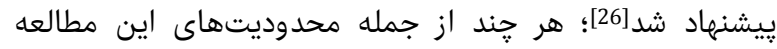

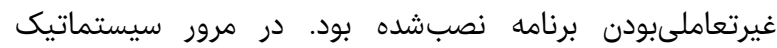

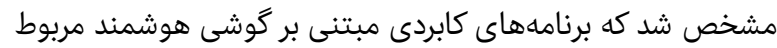

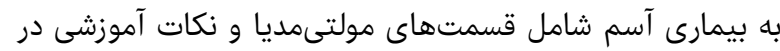

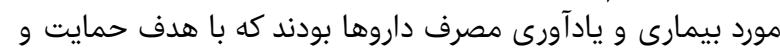

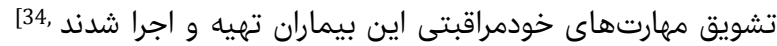

در اين مطالعه فقط ارزيابى اوليه نرمافزار صورت گرفت و در برد

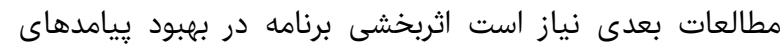

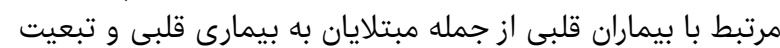

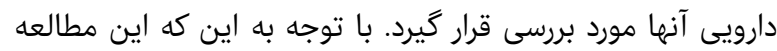

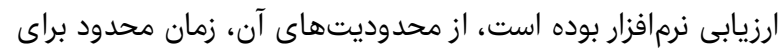

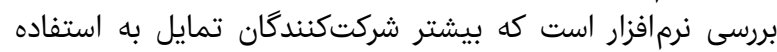

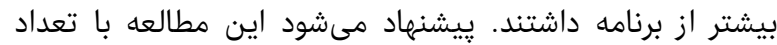

شركتكنندگان بيشتر و در بازههاى زمانى طولانى إنتر انجام شود.

\section{نتيجه}

استفاده از برنامه كاربردى مبتيرى مدئ بر گوشى هوشمند مىتواند در

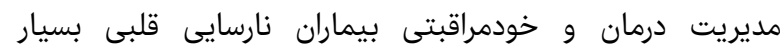
كمككننده و مفيد باشد.

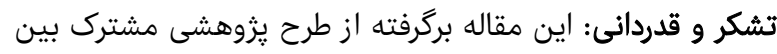

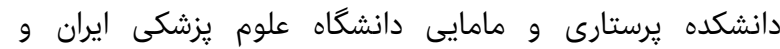

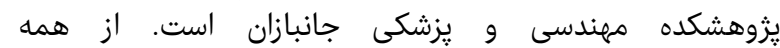

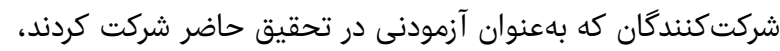

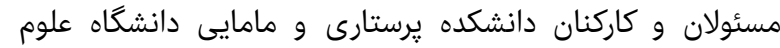

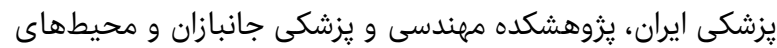

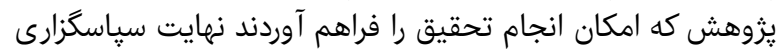

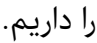

براساس مقياس سنجش برنامه كاربردى گوشى هوشمند (MARS)

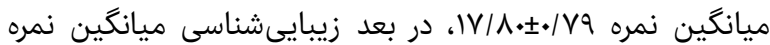

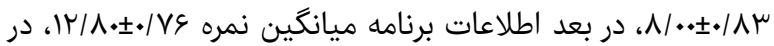

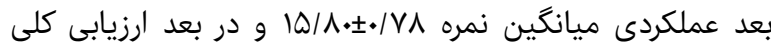

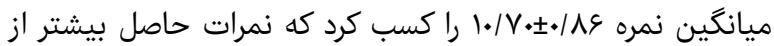

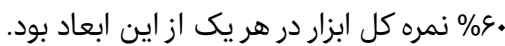

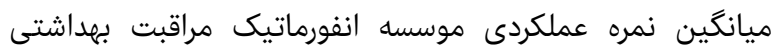
(IMS)

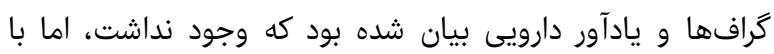

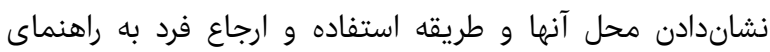

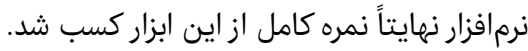

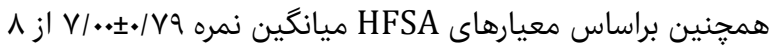
حاصل شد. تنها در يك مورد نبود محتواى آموزشى دري در مورد

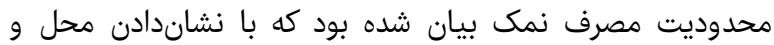

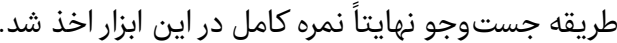

بحث

در اين مطالعه، برنامه كاربردى "قلب هوشمند من" طراحى شد.

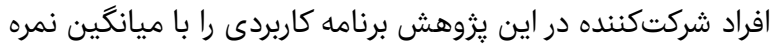

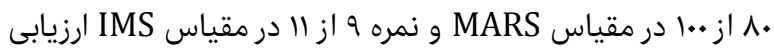

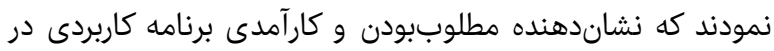

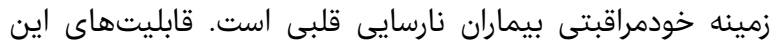

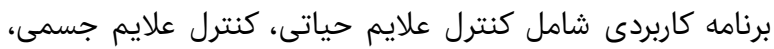

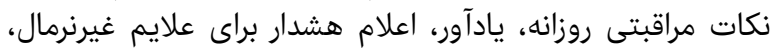

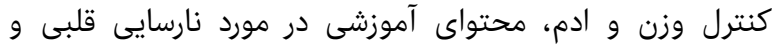
بيمارىهاى مزمن ديكر بود.

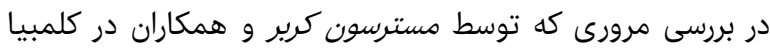

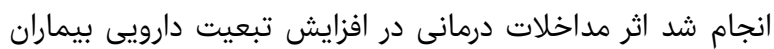

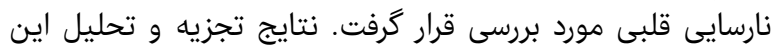

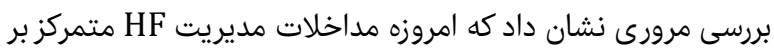

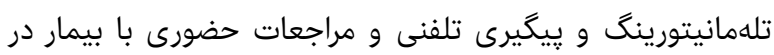

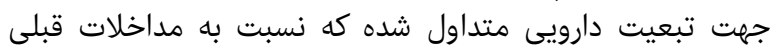

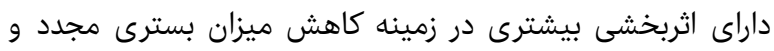

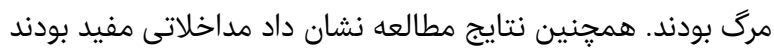

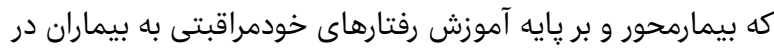

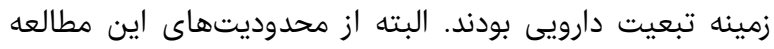

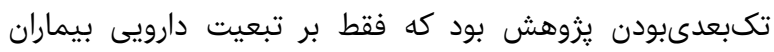

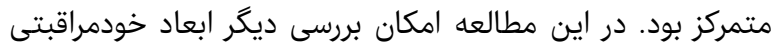

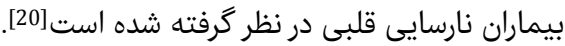

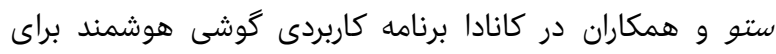

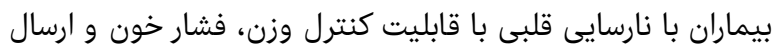

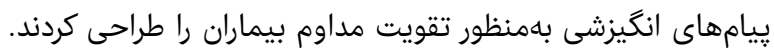

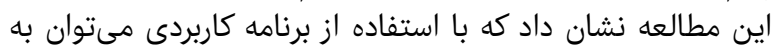

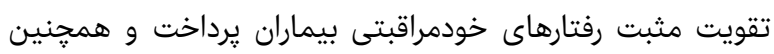

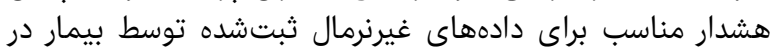

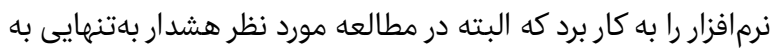

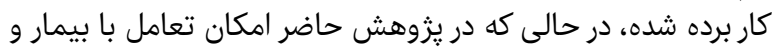

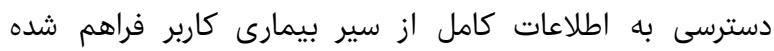

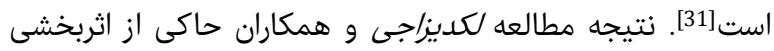

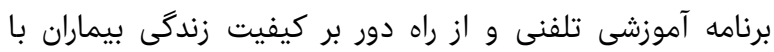

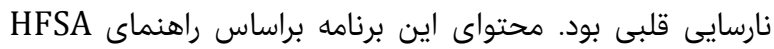

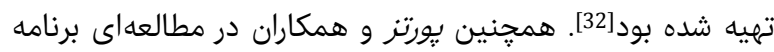


12- Free C, Phillips G, Watson L, Galli L, Felix L, Edwards $P$, et al. The effectiveness of mobile-health technologies to improve health care service delivery processes: a systematic review and meta-analysis. PLoS Med. 2013;10(1):e1001363.

13- Mirkovic J, Kaufman DR, Ruland CM. Supporting cancer patients in illness management: usability evaluation of a mobile app. JMIR Mhealth Uhealth. 2014;2(3):e33.

14- Lupton D. Quantifying the body: monitoring and measuring health in the age of mHealth technologies. Critical Public Health. 2013;23(4):393-403.

15- Chenari R, Noroozi A, Tahmasebi R. Relation between perceived social support and health promotion behaviors in chemical veterans in Ilam province on 2012-13. Iran J War Public Health. 2013;6(1):1-10. [Persian]

16- Vahedi M, Feyzi F, Ebadi A, Kachooei H. The effect of presence (lecture) and non-presence (booklet \& reminder) education method on nurse's knowledge, attitude and performance (kap) about autonomic dysreflexia in Tehran in 1389. Iran J War Public Health. 2012;4(4):21-8. [Persian]

17- Ahmadi M, Baha-al-din Beigi K, Nouri T. Provide telemedicine services to veterans. J Hosp. 2014;(Special):1-13. [Persian]

18- Zannad F, Gattis Stough W, Rossignol P, Bauersachs J, McMurray JJ, Swedberg K, et al. Mineralocorticoid receptor antagonists for heart failure with reduced ejection fraction: integrating evidence into clinical practice. Eur Heart J. 2012;33(22):2782-95.

19- Hale K, Capra S, Bauer J. A framework to assist health professionals in recommending high-quality apps for supporting chronic disease self-management: illustrative assessment of type 2 diabetes apps. JMIR Mhealth Uhealth. 2015;3(3):e87.

20- Masterson Creber RM, Maurer MS, Reading M, Hiraldo G, Hickey KT, Iribarren S. Review and analysis of existing mobile phone apps to support heart failure symptom monitoring and self-care management using the mobile application rating scale (MARS). JMIR Mhealth Uhealth. 2016;4(2):e74.

21- Nasi G, Cucciniello M, Guerrazzi C. The role of mobile technologies in health care processes: the case of cancer supportive care. J Med Internet Res. 2015;17(2):e26.

22- Martín JAC, Martínez-Pérez B, de la Torre-Díez I, López-Coronado M. Economic impact assessment from the use of a mobile app for the self-management of heart diseases by patients with heart failure in a Spanish region. J Med Syst. 2014;38(9):96.

23- Stevens DJ, Jackson JA, Howes N, Morgan J. Obesity surgery smartphone apps: a review. Obes Surg. 2014;24(1):32-6.

24- Wallace LS, Dhingra LK. A systematic review of smartphone applications for chronic pain available for download in the United States. J Opioid Manag. 2014;10(1):63-8.

25- Varnfield M, Karunanithi M, Lee CK, Honeyman E, Arnold D, Ding H, et al. Smartphone-based home care model improved use of cardiac rehabilitation in postmyocardial infarction patients: results from a randomised controlled trial. Heart. 2014;100(22):17709.

26- Jobrani H, Aghebati N, Shahriyari Sh, Behnam Vashani HR, Sehatbakhsh S, Esfahanizadeh J. Introduction and use of an education-notification application for patients undergoing heart valve

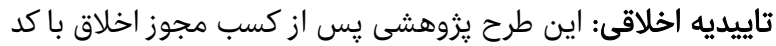

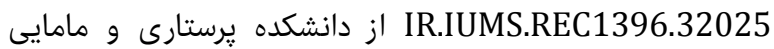

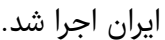
تعارض منافع: نويسندگًان مقاله هيج گَّنه تعارض منافعى ندارند.

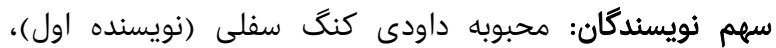

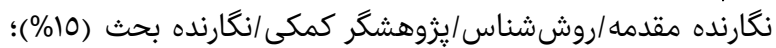

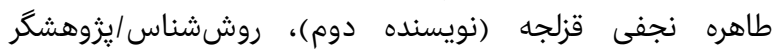

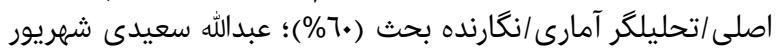

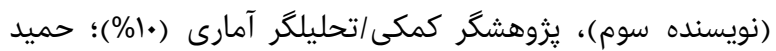

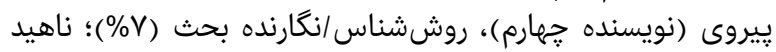

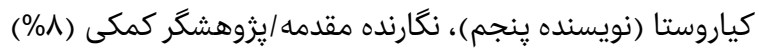

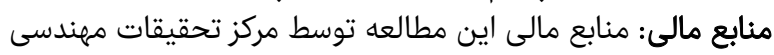

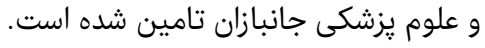

منابع

1- Mann DL, Zipes DP, Libby P, Bonow RO. Braunwald's heart disease: a textbook of cardiovascular medicine. 10th Edition. Philadelphia: Elsevier Saunders; 2014.

2- Newby LK. Understanding population cardiovascular health: harnessing the power of electronic health records. Circulation. 2015;132(14):1303-4.

3- Go AS, Mozaffarian D, Roger VL, Benjamin EJ, Berry JD, Blaha MJ, et al. Heart disease and stroke statistics-2014 update: a report from the American Heart Association. Circulation. 2014;129(3):e28-e292.

4- Baghianimoghadam MH, Shogafard G, Sanati HR, Baghianimoghadam B, Mazloomy SS, Askarshahi M. Application of the health belief model in promotion of self-care in heart failure patients. Acta Med Iran. 2013;51(1):52-8.

5- Costantino ME, Frey B, Hall B, Painter P. The influence of a postdischarge intervention on reducing hospital readmissions in a Medicare population. Popul Health Manag. 2013;16(5):310-6.

6- Fitzgerald AA, Powers JD, Ho PM, Maddox TM, Peterson PN, Allen LA, et al. Impact of medication nonadherence on hospitalizations and mortality in heart failure. J Card Fail. 2011;17(8):664-9.

7- Ruppar TM, Cooper PS, Mehr DR, Delgado JM, DunbarJacob JM. Medication adherence interventions improve heart failure mortality and readmission rates: systematic review and meta-analysis of controlled trials. J Am Heart Assoc. 2016;5(6). pii: e002606.

8- DeWalt DA, Schillinger D, Ruo B, Bibbins-Domingo K, Baker DW, Holmes GM, et al. A multisite randomized trial of a single- session versus multi-session literacy sensitive self-care intervention for patients with heart failure. Circulation. 2012;125(23):2854-62.

9- Heart Failure Society of America, Lindenfeld J, Albert NM, Boehmer JP, Collins SP, Ezekowitz JA, et al. HFSA 2010 comprehensive heart failure practice guideline. J Card Fail. 2010;16(6):e1-194.

10- Unverzagt S, Meyer G, Mittmann S, Samos FA, Unverzagt M, Prondzinsky R. Improving treatment adherence in heart failure: a systematic review and meta-analysis of pharmacological and lifestyle interventions. Dtsch Ärztebl Int. 2016;113(25):423-30.

11- Masterson Creber RM, Hickey KT, Maurer MS. Gerontechnologies for older patients with heart failure: What is the role of smartphones, tablets, and remote monitoring devices in improving symptom monitoring and self-care management? Curr Cardiovasc Risk Rep. 2016;10(10):30. 
ــ طراحى و ارزيابى برنامه كاربردى مبتنى بر گوشى هوشمند براى مديريت درمان افراد با نارسايى قلبى اسا

31- Seto E, Leonard KJ, Cafazzo JA, Barnsley J, Masino C, Ross HJ. Mobile phone-based telemonitoring for heart failure management: a randomized controlled trial. J Med Internet Res. 2012;14(1):e31.

32- Lakdizaji S, Hassankhni H, Agdam AM, Khajegodary M, Salehi R. Effect of educational program on quality of life of patients with heart failure: A randomized clinical trial. J Caring Sci. 2013;2(1):11-8.

33- Portz JD, Vehovec A, Dolansky MA, Levin JB, Bull S, Boxer R. The development and acceptability of a mobile application for tracking symptoms of heart failure among older adults. Telemed J E Health. 2018;24(2):161-5.

34- Marcano Belisario JS, Huckvale K, Greenfield G, Car J, Gunn LH. Smartphone and tablet self management apps for asthma. Cochrane Database Syst Rev. 2013;(11):CD010013.

35- Cooper RA. Surgical site infections: epidemiology and microbiological aspects in trauma and orthopaedic surgery. Int Wound J. 2013;10 Suppl 1:3-8. replacement. Evid Based Care J. 2016;6(3):31-6.

27- Stoyanov SR, Hides L, Kavanagh DJ, Zelenko O, Tjondronegoro D, Mani M. Mobile app rating scale: a new tool for assessing the quality of health mobile apps. JMIR Mhealth Uhealth. 2015;3(1):e27.

28- Aitken M, Gauntlett C. Patient apps for improved healthcare: from novelty to mainstream. Parsippany, NJ: IMS Institute for Healthcare Informatics; 2013.

29- Hunter WG, Kelly JP, McGarrah RW, Khouri MG, Craig D, Haynes C, et al. Metabolomic profiling identifies novel circulating biomarkers of mitochondrial dysfunction differentially elevated in heart failure with preserved versus reduced ejection fraction: evidence for shared metabolic impairments in clinical heart failure. J Am Heart Assoc. 2016;5(8):e003190.

30- Nocum AA, Baltao JM, Agustin DR, Portus AJ. Ergonomic evaluation and design of a mobile application for maternal and infant health for smartphone users among lower-income class Filipinos. Procedia Manuf. 2015;3:5411-8. 